2. O. Zariski and P. Samuel, Commutative algebra. Vol. 1, University Series in Higher Math., Van Nostrand, Princeton, N. J., 1958. MR 19, 833.

3. H. Bass, K-theory and stable algebra, Inst. Hautes Études Sci. Publ. Math. No. 22 (1964), 5-60. MR 30 \#485.

4. B. L. van der Waerden, Modern algebra. Vol. I, Springer, Berlin, 1930; English transl., Ungar, New York, 1949. MR 10, 587.

INSTITUTE OF MATHEMATICS, HEBREW UNIVERSITY OF JERUSALEM, JERUSA LEM, ISRAEL

PROCEEDINGS OF THE

AMERICAN MATHEMATICAL SOCIETY

Volume 50, July 1975

\title{
HOMOLOGICAL ALGEBRA IN TOPOI
}

\section{H. VAN OSDOL}

ABSTRACT. Let A be the category of sheaves of universal algebras of some fixed type on a Grothendieck topology. There is defined a cohomology theory $H^{*}$ such that if $A$ is an object in $\mathbf{A}$ and $M$ is an $A$-module then $H^{1}(A, M)$ is in one-to-one correspondence with the equivalence classes of singular extensions of $A$ by $M$. When $\mathbf{A}$ is the category of sheaves of $R$-modules for some sheaf of rings $R$, then $H^{n}(A, M) \cong$ $\operatorname{Ext}^{n}(A, M)$ for all $n \geq 0$.

The purpose of this note is to extend the results of [4] and [5] to general topoi, i.e. to sheaves on a Grothendieck topology. The extension has been made possible by the following theorem: Let $\mathbf{E}$ be a topos. Then there exists a complete Boolean algebra $B$ and a left exact cotriple $G$ on $\widetilde{\mathbf{B}}$ (= sheaves on $B$ for the canonical topology) such that $\mathbf{E}$ and $\widetilde{\mathbf{B}}_{G}$ (= the category of $G$-coalgebras) are equivalent categories.

Barr's proof of this theorem can be found in [1]. Lawvere, who conjectured the theorem, paraphrases it by saying that any topos has enough Boolean-valued points. The value of the theorem is that in $\widetilde{\mathbf{B}}$ subobjects have complements and epimorphisms split, i.e. $\widetilde{B}$ is very "set-like". One

Received by the editors March 1, 1974 and, in revised form, April 3, 1974. $18 \mathrm{G} 15$.

AMS (MOS) subject classifications (1970). Primary 18C15, 18F10; Secondary

Key words and phrases. Cotriple, topos, principal object, extension. 
uses this either indirectly or directly by transferring questions about $\mathbf{E}$ to questions about $\widetilde{\mathbf{B}}$. In this note we use the former approach.

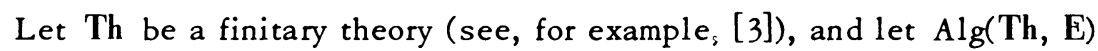
be the category of product preserving functors $\mathbf{T h} \rightarrow \mathbf{E}$. For example, when Th is the theory of abelian groups, $\operatorname{Alg}(\mathrm{Th}, \mathrm{E})$ is the category of abelian group objects in $\mathbf{E}$. Let

$$
\mathrm{E} \underset{Q}{\stackrel{S}{\rightleftarrows}} \widetilde{\mathbf{B}}
$$

be the adjoint pair arising from the Barr-Lawvere theorem, $U: A \lg (T h, \widetilde{B}) \rightarrow$ $\widetilde{\mathbf{B}}$ and $U^{\prime}: \operatorname{Alg}(\mathbf{T h}, \mathbf{E}) \rightarrow \mathbf{E}$ the usual "forgetful" functors. Since both $S$ and $Q$ preserve finite products, we get functors

$$
\operatorname{Alg}(\mathrm{Th}, \mathrm{E}) \stackrel{S^{\prime}}{\rightarrow} \quad \operatorname{Alg}(\mathrm{Th}, \widetilde{\mathbf{B}}) \stackrel{Q^{\prime}}{\rightarrow} \quad \operatorname{Alg}(\mathrm{Th}, \mathrm{E})
$$

via composition, e.g. given $A:$ Th $\rightarrow$ E we get $S^{\prime}(A)=$ Th $\stackrel{A}{\rightarrow} \mathbf{E} \stackrel{S}{\rightarrow} \widetilde{\mathbf{B}}$. An easy computation shows that $S^{\prime}$ is left adjoint to $Q^{\prime}$, and in fact that the cotriple $S^{\prime} Q^{\prime}$ is a lifting of $S Q=G$ to $\mathrm{Alg}(\mathrm{Th}, \widetilde{\mathbf{B}})$ in the sense of [6]. Moreover $U$ is tripleable (see [7] for the first proof, but a direct proof is not difficult to give).

Hence there is a mixed distributive law $\lambda: U F S Q \rightarrow S Q U F$ where $F$ is left adjoint to $U$ (see [6, pp. 235-236]) and a lifting of the triple $U F$ to a triple $T^{\prime}$ on E. Using the fact that $U S^{\prime}=S U^{\prime}$ one can show that $S^{\prime}$ is cotripleable and $U^{\prime}$ is tripleable. It follows that $\mathrm{Alg}(\mathrm{Th}, \mathrm{E})$ is bialgebraic over $\widetilde{\mathbf{B}}$ in the sense of [4], and hence that there is a cohomology theory, the first group of which classifies principal objects in $\operatorname{Alg}(\mathbf{T h}, \mathbf{E})$. For example, let $\mathrm{Th}$ be the theory of commutative rings, let $R$ be a Th-algebra (= a sheaf of commutative rings), let $M$ be an $R$-module ( $=$ a sheaf of $R$-modules) and

$$
C^{i, j}(R, M)=\operatorname{Der}_{R}\left(\left(F^{\prime} U^{\prime}\right)^{i+1} R,\left(Q^{\prime} S^{\prime}\right)^{j+1} M\right) .
$$

Then the first homology group of the double complex $C^{*}, *(R, M)$ is in one-toone correspondence with the set of equivalence classes of singular extensions of $R$ by $M$. The "local triviality" condition which must be met is guaranteed because epimorphisms split in $\widetilde{\mathbf{B}}$. Notice that this provides a theoretical solution to the problem posed by Grothendieck in [2]. See [4] for more details and more examples.

In case that $\mathbf{T h}=\mathbf{A b}$ is the theory of abelian groups we can classify $n$-fold extensions for each $n \geq 1$. Let $M, N$ be objects in $\operatorname{Alg}(\mathbf{A b}, \mathbf{E})$, let $G^{\prime}$ be the free abelian group cotriple lifted to $\mathrm{Alg}(\mathbf{A b}, \mathbf{E})$, and $T^{\prime}=Q^{\prime} S^{\prime}$ as above. Let 


$$
C^{i, j}=A \lg (\mathbf{A b}, \mathbf{E})\left(G^{\prime{ }^{+}+1} M, T^{\prime j+1} N\right)
$$

then the $n$th homology group of $C^{*, *}$ is in one-to-one correspondence with $\operatorname{Ext}^{n}(M, N)$, the set of equivalence classes of exact sequences $0 \rightarrow N \rightarrow$ $A_{0} \rightarrow \cdots \rightarrow A_{n-1} \rightarrow M \rightarrow 0$ in $A \lg (\mathrm{Ab}, \mathrm{E})$. The proof of this fact is identical with that given in [5]. The argument rests ultimately on the fact that epimorphisms split in $\widetilde{\mathbf{B}}$.

As Barr points out [1], the argument just outlined works equally well for sheaves of $R$-modules where $R$ is a sheaf of rings. Perhaps a few words about the proof are in order. Since $Q S(R \times R)$ is naturally isomorphic to $Q S R \times Q S R$, it follows that the adjunction $\eta R: R \rightarrow Q S R$ is a ring homomorphism. Thus if $N$ is a sheaf of $R$-modules then $\eta R$ makes $Q S N$ into a sheaf of $R$-modules in such a way that $\eta N: N \rightarrow Q S N$ is an $R$-homomorphism. By induction, $(Q S)^{i} N$ is a sheaf of $R$-modules and each $(Q S)^{i} \eta(Q S)^{n-i} N$ is an $R$-homomorphism. Letting $G$ ' be the "free sheaf of $R$-modules" cotriple, we can proceed as in the last paragraph by letting $C^{i, j}=\operatorname{Hom}_{R}\left(G^{\prime i+1} M,(Q S)^{j+1} N\right)$. Then the $n$th homology group of $C^{*}{ }^{*}$ is in one-to-one correspondence with $\operatorname{Ext}_{R}^{n}(M, N)$.

\section{REFERENCES}

1. M. Barr, Toposes without points (to appear).

2. A. Grothendieck, Catégories cofibrées additives et complexe cotangent relatif, Lecture Notes in Math., no. 79, Springer-Verlag, Berlin and New York, 1968. MR 39 \# 2835.

3. B. Pareigis, Kategorien und Funktoren, Mathematische Leitfäden, Teubner, Stuttgart, 1969; English transl., Pure and Appl. Math., vol. 39, Academic Press, New York, 1970. MR $42 \# 337 \mathrm{a}, \mathrm{b}$.

4. D. Van Osdol, Bicohomology theory, Trans. Amer. Math. Soc. 183 (1973), $449-476$.

5. - Remarks concerning $\operatorname{Ext}^{*}(M,-)$, Bull. Amer. Math. Soc. 76 (1970), 612-617. MR 42 \#6081.

6. - "Sheaves in regular categories", in M. Barr et al., Exact categories and categories of sheaves, Lecture Notes in Math., no. 236, Springer-Verlag, Berlin and New York, 1971.

7. H. Wolff, V-localizations and V-triples, Dissertation, University of Illinois, Urbana, Ill., 1970.

DEPARTMENT OF MATHEMATICS, UNIVERSITY OF NEW HAMPSHIRE, DURHAM, NEW HAMPSHIRE 03824 